\title{
Evaluation of the Association between Neck Pain and the Trapezius Muscles in Patients with Cervical Myelopathy Using Motor Evoked Potential: A Retrospective Study
}

\author{
Sadayuki Ito ${ }^{1}$, Yoshihito Sakai ${ }^{2}$, Atsushi Harada ${ }^{2}$, Kei Ando ${ }^{1}$, Kazuyoshi Kobayashi ${ }^{1}$, \\ Hiroaki Nakashima ${ }^{1}$, Masaaki Machino ${ }^{1}$, Shunsuke Kambara ${ }^{1}$, Taro Inoue ${ }^{1}$, \\ Tetsuro Hida ${ }^{3}$, Kenyu Ito $^{4}$, Naoki Ishiguro ${ }^{1}$, Shiro Imagama ${ }^{1}$ \\ ${ }^{I}$ Department of Orthopedic Surgery, Nagoya University Graduate School of Medicine, Nagoya, Japan \\ ${ }^{2}$ Department of Orthopedic Surgery, National Center for Geriatrics and Gerontology, Obu, Japan \\ ${ }^{3}$ Department of Orthopedic Surgery, Nagoya Daini Red Cross Hospital, Nagoya, Japan \\ ${ }^{4}$ Department of Orthopedic Surgery, Konan Kosei Hospital, Konan, Japan
}

Study Design: Retrospective study.

Purpose: We aimed to use motor evoked potentials (MEPs) to examine the association of electrophysiological assessment of the trapezius muscle with neck pain.

Overview of Literature: Previous reports on the association of neck pain with the trapezius muscle have focused on surface electromyograms and muscle oxygenation; however, to our knowledge, none of these studies included detailed data on MEPs.

Methods: The study included 100 patients with cervical myelopathy who underwent surgery at the National Center for Geriatrics and Gerontology in Obu, Japan from June 2010 to March 2013. Before the surgery, neck pain was evaluated using a Visual Analog Scale (a score $\geq 50$ indicated neck pain and a score $<50$ indicated no neck pain). The preoperative cross-sectional areas of the trapezius muscles were measured with cervical magnetic resonance imaging sagittal T2-weighted images. Cranial stimulation under general anesthesia was used to derive the MEPs, enabling the measurement of latency and amplitude, using preoperative MEPs of the trapezius muscles.

Results: The MEP of the trapezius muscle in patients with neck pain had significantly shorter latencies than those in patients who did not have neck pain. However, there was no significant difference in the amplitude between patients with and without neck pain. However, this tended to be greater in patients with neck pain as compared to that in those without neck pain. The cross-sectional area of the trapezius muscle in patients with neck pain was significantly smaller than that in those who did not have neck pain. Conclusions: MEPs revealed electrophysiological abnormalities of the trapezius muscles in patients with neck pain, supporting a relationship of neck pain with the trapezius muscles.

Keywords: Trapezius muscle; Neck pain; Cervical myelopathy; Motor evoked potentials; Cross-sectional areas

Received Apr 22, 2020; Revised May 20, 2020; Accepted May 22, 2020

Corresponding author: Shiro Imagama

Department of Orthopedic Surgery, Nagoya University Graduate School of Medicine, 65 Tsurumai-cho, Showa-ku, Nagoya 466-8560, Japan

Tel: +81-52-741-2111, Fax: +81-52-744-2260, E-mail: imagama@med.nagoya-u.ac.jp 


\section{Introduction}

Causes of neck pain include impaired nerve roots, intervertebral disks, facet joints, and muscles [1]. Muscles are more important for the stability of the cervical vertebrae as compared to the intervertebral disks [2]. Furthermore, neck pain is often seen in people who spend long periods in the same position while performing deskwork [3]. In terms of stability of the cervical vertebrae, the neck extensor muscles are believed to be important in the prevention of bending and rotation [1]. Within the neck muscles, there are deep muscles, such as the multifidus, cervical semi-spinal, semispinalis, and splenius muscles, and superficial muscles, such as the trapezius muscles. Many studies have reported on the relationship between the deep muscles and the trapezius muscles [4-8]. Moreover, other studies have used surface electromyography (EMG) to analyze the relationship between neck pain and the trapezius muscles; however, surface EMG results are influenced by physiological reactions resulting from stress, installation of the EMG equipment, posture, and test environment, leading to low reproducibility of EMG results $[8,9]$.

Many studies have focused on people with neck pain [19] and investigated the factors associated with postoperative neck pain in people with cervical myelopathy $[10,11]$. However, few reports have investigated preoperative neck pain in people with cervical myelopathy. Therefore, we designed this study to investigate the relationship between neck pain and the trapezius muscles preoperatively in patients with cervical myelopathy, using motor evoked potential (MEP) under intraoperative anesthesia. The methods of this study enabled us to conduct a detailed and accurate electrophysiological test that was not influenced by physiological reactions related to stress and test environment.

\section{Materials and Methods}

\section{Participants}

Consecutive patients undergoing cervical laminoplasty for cervical myelopathy were enrolled between June 2010 and March 2013. Spine surgeons at the National Center for Geriatrics and Gerontology determined the surgical indications as per the symptoms and medical imaging results of patients with cervical myelopathy. The surgery was performed at the level of $\mathrm{C} 3-\mathrm{C} 7$ where stenosis was noted. C3-C6, C3-C7, C4-C6, and C4-C7 laminoplasty were included. Those who had undergone prior cervical spine surgery were excluded; further, those for whom the MEP control wave could not be recorded, Visual Analog Scale (VAS) scores could not be determined, magnetic resonance imaging (MRI) data were unavailable, or an assessment of muscle hardness could not be performed were also excluded.

We conducted this study as per the principles of the Helsinki Declaration. This study was conducted with the approval of the Research Ethics and Conflicts Committee of the National Center for Geriatrics and Gerontology of Japan (approval no., 433). All the participants provided informed consent for study participation.

\section{Measurements}

We evaluated neck pain using the VAS, with a score $\geq 50$ indicating neck pain and a score $<50$ indicating no neck pain $[12,13]$. To measure the MEPs of the trapezius muscles, we performed transcranial stimulation using a D185 MultiPulse stimulator (Digitimer Ltd., Tokyo, Japan). The stimulation parameters were as follows: five consecutive stimuli with $2 \mathrm{~ms}$ interstimulus intervals; 500 V; a 50-1,000-Hz filter; and 100 ms epoch time, with $\leq 20$ individual recorded responses. The stimulated point was $2 \mathrm{~cm}$ anterior and $6 \mathrm{~cm}$ lateral to the $\mathrm{Cz}$ location (International 10-20 System) over the cerebral cortex motor area. Using the Neuromaster MEE-1200 series (Nihon Kohden, Tokyo, Japan), MEPs were recorded from the bilateral trapezius muscles. For derivation of the compound action potential of the trapezius muscles, we used needle electrodes with the electrodes located at the midpoint between the 7th cervical spinous process and the acromion. We measured the latency and amplitude using a control waveform that was measured preoperatively to exclude the influence of surgery.

The drugs that were initially administered included propofol $(3-4 \mu \mathrm{g} / \mathrm{mL})$, fentanyl $(2 \mu \mathrm{g} / \mathrm{kg})$, and vecuronium $(0.12-0.16 \mathrm{mg} / \mathrm{kg})$. Anesthesia was maintained using propofol $(3 \mu \mathrm{g} / \mathrm{mL})$, fentanyl $(1-2.5 \mu \mathrm{g} / \mathrm{kg} / \mathrm{hr})$, and vecuronium (0.01-0.04 mg/kg/hr). Benzodiazepines were administered as a pre-anesthetic medication; however, the use was limited or avoided because it suppresses the latency and amplitude. Our participants were maintained under normothermia, and the anesthetist attempted to 
maintain end-tidal $\mathrm{CO}_{2}$ in the normal range throughout the surgery duration [14].

We assessed the trapezius muscle mass using a $1.5 \mathrm{~T}$ MRI scanner. Axial T2-weighted slices at the C3-C4, C4-C5, C5-C6, and C6-C7 intervertebral levels were obtained to measure the cross-sectional area (CSA) of the trapezius muscles. The trapezius muscle CSAs obtained from the right and left sides were combined for each participant. The CSAs of the muscles were measured with area calculation software (SYNAPSE; Fujifilm Medical, Tokyo, Japan). CSA measurements were performed by one investigator (S.I.) who manually traced the defined region of interest within the fascial border of each muscle, bilaterally, on the T2-weighted images (Fig. 1). The measurement was performed 2 times, and the obtained values

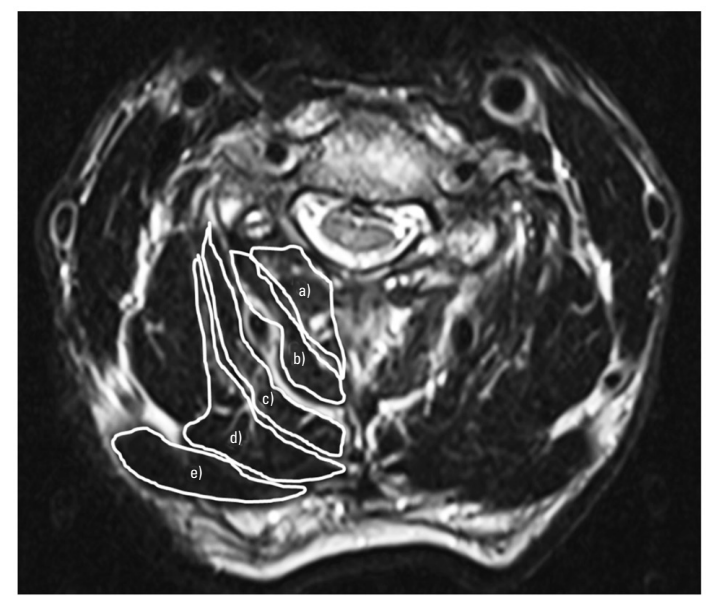

Fig. 1. Using preoperative magnetic resonance imaging, the cross-sectional areas of the trapezius muscles at the C3-4, C4-5, C5-6, and C6-7 intervertebral levels were measured on T2-weighted axial images. ${ }^{\text {al }}$ Multifidus, ${ }^{\text {bl }}$ semispinalis cervicis, ${ }^{c /}$ semispinalis capitis, ${ }^{\mathrm{d}}$ splenius capitis, and ${ }^{\mathrm{el}}$ trapezius. were averaged. The intra- and interobserver errors were calculated as follows: five randomly chosen magnetic resonance images were measured independently by one investigator (S.I.) and then measured again by a second investigator (Y.S.) [15].

\section{Statistical analyses}

Participant demographics, latency and amplitude of the trapezius muscle MEPs, and the muscle mass of the trapezius muscles were compared between patients with neck pain and those without neck pain with chi-square test or Student $t$-test, as appropriate. Pearson's correlation coefficient was used to assess the relationship between the muscle mass of the trapezius muscles and the latency and amplitude of the trapezius muscle MEPs. The data were aggregated and analyzed using IBM SPSS statistics ver. 22.0 software (IBM Corp., Armonk, NY, USA), with $p<0.05$ indicating statistical significance.

\section{Results}

We enrolled 100 participants who had undergone surgery for cervical myelopathy from June 2010 to March 2013. The average patient age at the time of surgery was 72.2 \pm 8.7 years. Twenty-seven patients underwent C3C6 laminoplasty, 37 underwent C3-C7 laminoplasty, 10 underwent C4-C6 laminoplasty, and 26 underwent C4C7 laminoplasty. There were 31 participants in the neck pain group and 69 subjects in the no neck pain group. The sex, age, height, body weight, range of motion, and cervical lordosis of the groups were not significantly different (Table 1).

Table 1. Demographic and clinical characteristics of patients with cervical myelopathy

\begin{tabular}{lccc} 
Characteristic & Neck pain $(+)($ VAS score $\geq 50)$ & Neck pain $(-)$ (VAS score $<50)$ & $p$-value \\
No. of patients & 31 & $71.6 \pm 8.8$ & 0.274 \\
\hline Age $(\mathrm{yr})$ & $73.8 \pm 9.8$ & $50: 19$ & 0.529 \\
\hline Male:female & 22.9 & $159.3 \pm 9.0$ & 0.132 \\
\hline Height $(\mathrm{cm})$ & $156.3 \pm 9.9$ & $61.4 \pm 11.0$ & 0.336 \\
\hline Weight $(\mathrm{kg})$ & $59.1 \pm 10.0$ & $33.6 \pm 13.7$ & 0.903 \\
\hline Range of motion $\left({ }^{\circ}\right)(\mathrm{X}-\mathrm{p})$ & $33.2 \pm 13.6$ & $13.0 \pm 11.0$ & 0.435 \\
\hline Cervical lordotic angle $\left(^{\circ}\right)(\mathrm{X}-\mathrm{p})$ & $15.0 \pm 13.0$ & & \\
\hline
\end{tabular}

Values are presented as number or mean \pm standard deviation for each group. Range of motion: C2-7 lordotic angle of extension to C2-7 lordotic angle of flexion (lateral view); cervical lordotic angle: C2-7 lordotic angle (lateral view, neutral position).

VAS, Visual Analog Scale. 
Table 2. Comparison of the latency and amplitude of preoperative MEP and cross-sectional area of the trapezius muscle between patients with and without neck pain

\begin{tabular}{lccc} 
Trapezius muscle & Neck pain $(+)($ VAS score $\geq 50)$ & Neck pain $(-)($ VAS score $<50)$ & $p$-value \\
\hline Latency $(\mathrm{MEP})(\mathrm{ms})$ & $23.21 \pm 2.45$ & $24.76 \pm 2.80$ & 0.009 \\
Amplitude $(\mathrm{MEP})(\mu \mathrm{V})$ & $2,355 \pm 675$ & $2,003 \pm 900$ & 0.055 \\
Muscle cross-sectional area $\left(\mathrm{mm}^{2}\right)$ & $2,922 \pm 470$ & $3,334 \pm 625$ & 0.001 \\
\hline
\end{tabular}

Values are presented as mean \pm standard deviation for each group.

MEP, motor evoked potential; VAS, Visual Analog Scale.

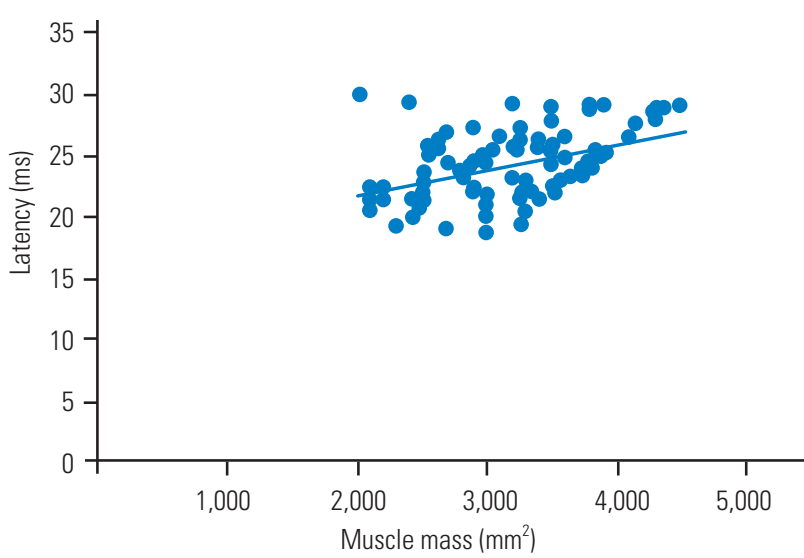

Fig. 2. The association between the latency of preoperative motor evoked potentials and cross-sectional area of the trapezius muscle $(r=0.477, p<0.001)$.

Latency was significantly shorter in the neck pain group as compared to that in the no neck pain group (neck pain group, 23.21 \pm 2.45 ; no neck pain group, $24.76 \pm 2.80$; $p<0.05)$ (Table 2). There was no significant difference in the amplitudes; however, the amplitude tended to be larger in patients with neck pain (neck pain group, 2,356 \pm 675 ; no neck pain group, $2,015 \pm 939 ; p=0.071$ ) (Table 2).

The CSAs of the trapezius muscles were significantly smaller in those with neck pain than in those without neck pain (neck pain group, $2,922 \pm 470 \mathrm{~mm}^{2}$; no neck pain group, 3,334 $\pm 625 \mathrm{~mm}^{2} ; p=0.001$ ) (Table 2). Furthermore, the CSAs of the trapezius muscles were significantly different; therefore, we examined the relationship between trapezius muscle mass and MEP and found a positive correlation between the trapezius muscle mass and latency (Fig. 2) and a negative correlation between trapezius muscle mass and amplitude (Fig. 3).

\section{Discussion}

This study assessed the relationship between the trapezius muscles and neck pain in patients with cervical myelopathy using exercise-induced potential obtained under gen-

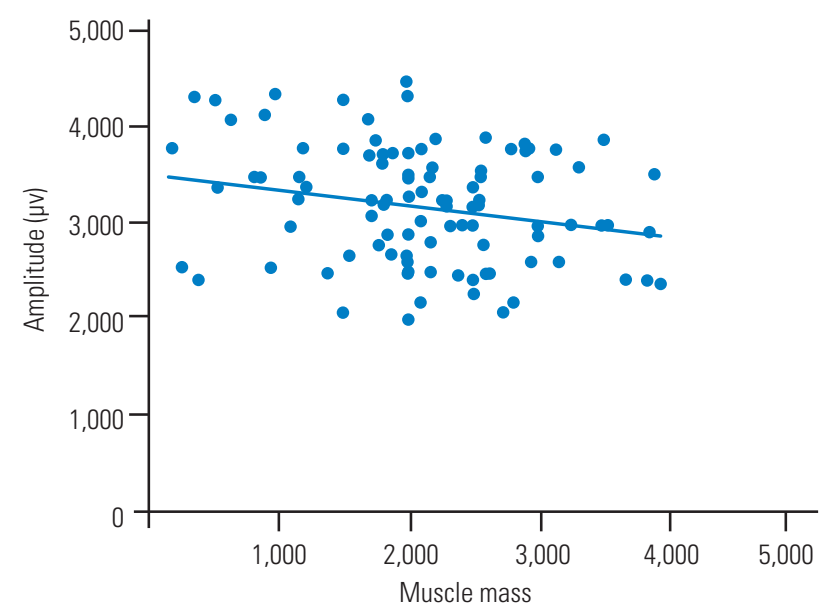

Fig. 3. The association between the amplitude of preoperative motor evoked potentials and cross-sectional area of the trapezius muscle $(r=-0.228, p=0.022)$.

eral anesthesia. Thus, we believe that our results are objective.

We observed reduced latency of the trapezius muscle MEPs. Although there was no significant difference in the amplitude, an increasing tendency was noted. In previous reports, the latency of the trapezius muscle MEPs was reportedly shorter and the amplitude was larger during spontaneous contraction compared to that at rest $[16,17]$. These findings suggest that in people with cervical myelopathy, the electrophysiological status of the trapezius muscles, when associated with neck pain, is comparable to that in those without cervical myelopathy.

Similar to previous reports [5], our study showed that the neck pain group had significantly smaller CSAs of the trapezius muscles, suggesting that atrophy of the trapezius muscles may cause neck pain. Moreover, a previous report that used surface EMG associated neck pain with electrophysiological abnormalities [6]. This finding was consistent with that in our study, wherein we observed a significantly shorter MEP latency in the neck pain group and a tendency toward increased amplitudes, suggesting increased electrophysiological activation of the trapezius 
muscles in individuals who had neck pain.

In our study, we observed a correlation between the latency and amplitude of MEP and trapezius muscle mass, irrespective of neck pain. Normally, if muscle atrophy occurs, an increase in the mobilization and firing rates of the motor units is required to maintain muscle tension, increasing the amplitudes of the EMG. Furthermore, based on the results of reduced MEP latency and increased amplitude after trapezius muscle atrophy and delayed latency in cases of spinal damage, it may be inferred that the atrophy causes poor function, leading to muscle contraction and ischemia that causes neck pain [18].

Studies conducted on monkeys and cats have reported several descending volleys occurring at 1-2 ms intervals in the pyramidal tract after cerebral cortex stimulation; thus, the same results are expected in humans. The reactive latency of the cortex is reduced during voluntary muscle contraction as compared to that at rest. When at rest, the membrane potential of most anterior horn cells is lower by 5-6 $\mathrm{mV}$ below the threshold; therefore, the anterior horn cells are not excited unless multiple descending volleys are elicited, with firing being impossible with the first descending volley, while that seeming to occur at the second and third descending volleys. However, during voluntary muscle contraction, the membrane potential of the anterior horn cells is almost at the threshold, making it possible to fire at the first descending volley. Therefore, there is an approximately 2-ms reduction in the latency during voluntary contraction than at rest [19]. In this study, an average reduction of $1.4 \mathrm{~ms}$ was noted in those with neck pain as compared to that in those without neck pain. Although the difference in the latency between voluntary contraction and that at rest was not significant, the membrane potential of the anterior horn cells may have been higher in those with neck pain. One possible factor for the increased membrane potential in the anterior horn cells is an abnormality in the descending control from the upper central nervous system. In this case, it is possible that this would causes an abnormality in the descending control of sensation [20-22], and an abnormality in the upper central nervous central could simultaneously cause myotonia and pain. Another possible factor is the increased excitability of the anterior horn cells that may affect latency reduction. Alternatively, in patients with neck pain, the muscle tone may be abnormally elevated, decreasing the MEP threshold and shortening the latency.

This study has certain limitations, including the influ- ence of the anesthetic on the MEP wavelengths because complete withholding of the anesthetic could not be achieved. However, an intraoperative total propofol dose $>1,550 \mathrm{mg}$ is reported to influence the waveform [23]; therefore, we believe that the influence of the anesthetic on the waveforms in our study was minimal. The innervation of the trapezius muscle is $\mathrm{C} 3$ or $\mathrm{C} 4$ and the MEP of trapezius muscle may be affected by stenosis at $\mathrm{C} 2 / 3$ and C3/4; however, we did not investigate the effect of stenosis in that area in the present study. Moreover, the participants were limited to people with neck pain who had indications for cervical myelopathy surgery. These results may not be applicable to individuals who had neck pain but did not have cervical myelopathy. Despite these limitations, our results suggest that in people with cervical myelopathy, poor muscle function can be caused by trapezius muscle atrophy leading to muscle contraction from a state of rest. Based on the reduced latency in the MEP, the possibility that myotonia and pain are caused by the central nervous system cannot be ruled out. Therefore, we believe that further investigations are needed.

\section{Conclusions}

Thus, in people with cervical myelopathy and neck pain, the trapezius muscle mass is lower and the MEP latency is shorter. Although no significant difference was observed in the MEP amplitude, it tended to be larger in people with neck pain. These observations suggest a correlation of neck pain with the trapezius muscles.

\section{Conflict of Interest}

No potential conflict of interest relevant to this article was reported.

\section{Acknowledgments}

The authors would like to thank the medical engineering division of the National Center for Geriatrics and Gerontology for its technical support.

\section{Author Contributions}

S. Ito conceived and designed the study; S. Ito, YS, and AH performed the research; all authors analyzed the data; YS contributed new methods and models; S. Ito wrote the 
paper; and all authors approved the final version of the manuscript.

\section{References}

1. Herkowitz HN, Garfin SR, Eismont FJ, Bell GR, Balderston RA. Rothman-Simeone, the spine. 5th ed. Philadelphia (PA): Saunders Elsevier; 2006.

2. Panjabi MM, Cholewicki J, Nibu K, Grauer J, Babat LB, Dvorak J. Critical load of the human cervical spine: an in vitro experimental study. Clin Biomech (Bristol, Avon) 1998;13:11-7.

3. Tornqvist EW, Kilbom A, Vingard E, et al. The influence on seeking care because of neck and shoulder disorders from work-related exposures. Epidemiology 2001;12:537-45.

4. Westgaard RH, Mork PJ, Loras HW, Riva R, Lundberg U. Trapezius activity of fibromyalgia patients is enhanced in stressful situations, but is similar to healthy controls in a quiet naturalistic setting: a case-control study. BMC Musculoskelet Disord 2013;14:97.

5. Zhang P, Shen Y, Zhang YZ, Ding WY, Xu JX, Cao JM. Preserving the C7 spinous process in laminectomy combined with lateral mass screw to prevent axial symptom. J Orthop Sci 2011;16:492-7.

6. Juul-Kristensen B, Clausen B, Ris I, et al. Increased neck muscle activity and impaired balance among females with whiplash-related chronic neck pain: a cross-sectional study. J Rehabil Med 2013;45:376-84.

7. Park SY, Yoo WG. Effect of sustained typing work on changes in scapular position, pressure pain sensitivity and upper trapezius activity. J Occup Health 2013;55:167-72.

8. Persson AL, Hansson GA, Kalliomaki A, Moritz U, Sjolund BH. Pressure pain thresholds and electromyographically defined muscular fatigue induced by a muscular endurance test in normal women. Clin J Pain 2000;16:155-63.

9. Bech KT, Larsen CM, Sjogaard G, Holtermann A, Taylor JL, Sogaard K. Voluntary activation of the trapezius muscle in cases with neck/shoulder pain compared to healthy controls. J Electromyogr Kinesiol 2017;36:56-64.

10. Stephens BF, Rhee JM, Neustein TM, Arceo R. Laminoplasty does not lead to worsening axial neck pain in the properly selected patient with cervical myelop- athy: a comparison with laminectomy and fusion. Spine (Phila Pa 1976) 2017;42:1844-50.

11. Kimura A, Shiraishi Y, Inoue H, Endo T, Takeshita K. Predictors of persistent axial neck pain after cervical laminoplasty. Spine (Phila Pa 1976) 2018;43:10-5.

12. Hjermstad MJ, Fayers PM, Haugen DF, et al. Studies comparing Numerical Rating Scales, Verbal Rating Scales, and Visual Analogue Scales for assessment of pain intensity in adults: a systematic literature review. J Pain Symptom Manage 2011;41:1073-93.

13. Given B, Given CW, Sikorskii A, et al. Establishing mild, moderate, and severe scores for cancer-related symptoms: how consistent and clinically meaningful are interference-based severity cut-points? J Pain Symptom Manage 2008;35:126-35.

14. Kobayashi K, Ando K, Yagi H, et al. Efficacy of anal needle electrodes for intraoperative spinal cord monitoring with transcranial muscle action potentials. Asian Spine J 2018;12:662-8.

15. Okada E, Matsumoto M, Ichihara D, et al. Crosssectional area of posterior extensor muscles of the cervical spine in asymptomatic subjects: a 10-year longitudinal magnetic resonance imaging study. Eur Spine J 2011;20:1567-73

16. Valls-Sole J, Pascual-Leone A, Brasil-Neto JP, Cammarota A, McShane L, Hallett M. Abnormal facilitation of the response to transcranial magnetic stimulation in patients with Parkinson's disease. Neurology 1994;44:735-41.

17. Van den Bos MA, Geevasinga N, Menon P, Burke D, Kiernan MC, Vucic S. Physiological processes influencing motor-evoked potential duration with voluntary contraction. J Neurophysiol 2017;117:1156-62.

18. Kosek E, Ekholm J, Hansson P. Modulation of pressure pain thresholds during and following isometric contraction in patients with fibromyalgia and in healthy controls. Pain 1996;64:415-23.

19. Jankowska E, Padel Y, Tanaka R. Disynaptic inhibition of spinal motoneurones from the motor cortex in the monkey. J Physiol 1976;258:467-87.

20. Suzuki T, Bunno Y, Onigata C, Tani M, Uragami S. Excitability of spinal neural function during several motor imagery tasks involving isometric opponens pollicis activity. NeuroRehabilitation 2013;33:171-6.

21. Luft AR, Skalej M, Stefanou A, Klose U, Voigt K. Comparing motion- and imagery-related activation in the human cerebellum: a functional MRI study. 
Hum Brain Mapp 1998;6:105-13.

22. Lotze M, Montoya P, Erb M, et al. Activation of cortical and cerebellar motor areas during executed and imagined hand movements: an fMRI study. J Cogn Neurosci 1999;11:491-501.
23. Ushirozako H, Yoshida G, Kobayashi S, et al. Impact of total propofol dose during spinal surgery: anesthetic fade on transcranial motor evoked potentials. J Neurosurg Spine 2019 Feb 8 [Epub]. https://doi. org/10.3171/2018.10.SPINE18322. 